\title{
A Wignerfunction Approach to Phonon Scattering
}

\author{
FLORIAN FROMMLET ${ }^{\mathrm{a}, *}$, PETER A. MARKOWICH ${ }^{\mathrm{b}, \dagger}$ and CHRISTIAN RINGHOFER ${ }^{\mathrm{c}, \ddagger}$ \\ ${ }^{\mathrm{a}}$ FB Mathematik, TU Berlin, Straße des 17. Juni 136, D-10623 Berlin, Germany; ${ }^{\mathrm{b}}$ Institute for Analysis and Numerics, \\ Altenberger Straße 69, A-4040 Linz, Austria; ${ }^{\mathrm{c}}$ Department of Mathematics, Arizona State University, \\ Tempe, Az 85287-1804, USA
}

(Received 13 August 1997; In final form 1 December 1998)

\begin{abstract}
We consider the motion of a single electron under phonon scattering caused by a crystal lattice. Starting from the Fröhlich Hamiltonian in the second quantization formalism we derive a kinetic transport model by using the Wigner transformation. Under the assumption of small electron-phonon interaction we derive asymptotically the operator representing electron-phonon scattering in the Wigner equation. We then consider some scaling limits and finally we give the connection of our result to the well known BarkerFerry equation.
\end{abstract}

Keywords: Phonon scattering, Wigner transformation, second quantization, weak interaction asymptotics

\section{INTRODUCTION}

If a semiconductor is modeled as a perfect crystal, the electrons moving in the crystal are not scattered by the lattice atoms at all. Because of thermal energy the atoms do not remain stationary but each atom moves in a region of space centered at its lattice point. The strong forces which are provided by the interaction of an atom with all the other atoms act on this atom when it is not at its lattice point. This leads to lattice vibrations which can be approximated by harmonic oscillations.
The independent normal modes of these oscillations are called phonons which can be considered as particles (bosons, $c f$. [7, 9]).

Two interaction processes occur: the electron can be scattered such that either a phonon is emitted or a phonon is absorbed, where in both processes the total wave number remains constant. Due to these scattering events the number of phonons is not conserved.

To deal with this non-constant number of particles one uses the procedure of 2 nd quantization, which was originally introduced in quantum

*Corresponding author. e-mail: frommlet@math.tu-berlin.de

†e-mail: marko@majestix.numa.uni-linz.ac.at

łe-mail: ringhofer@asu.edu 
field theory. So although our model is purely nonrelativistic we use the formalism of field quantization [3].

For the sake of simplicity we use a one-electron model which means that we neglect electronelectron interactions and are just interested in the dynamics of one electron. We will also neglect the effects of the periodical potential of the stationary lattice thus obtaining the so called Fröhlich Hamiltonian. Moreover we model the phonon Hamiltonian such that it has states of thermal equilibrium.

In this paper we will start with the von-Neumann equation for the density matrix and obtain (by applying the Wigner transform) a kinetic pseudodifferential equation for the Wigner-matrix. By taking the trace over the space of the phonons we are led to a quantum-transport equation for the Wignerfunction of the electron with a scattering term which still depends on certain elements of the Wigner matrix (not only on its phonon trace).

To obtain a "closed" equation for the Wignerfunction we assume that the interaction between the electrons and phonons is weak and we apply methods of asymptotic analysis (small coupling parameter). In the limit (no interaction at all) the phonons are assumed to be in a state of thermal equilibrium. Doing so we can finally derive a transport equation with a scattering term describing the electron-phonon interaction.

In chapter 4 we discuss some scaling limits of the derived scattering term and in the last chapter we present a new derivation of the Barker-Ferry equation.

\section{THE MATHEMATICAL MODEL}

In the 2nd quantization formulation we consider a modified version of the Fröhlich-Hamiltonian

$$
H=H_{e}+H_{p}+H_{e-p},
$$

where $H_{e}$ is the Hamiltonian for the free electron, $H_{p}$ for the free phonons and $H_{e-p}$ describes the interaction between the electron and the phonons. The Hamiltonian acts on wave-functions which lie in the state space

$$
\mathcal{S}=L^{2}\left(\mathbb{R}_{x}^{3}\right) \otimes \mathcal{F}_{S}
$$

where $\mathcal{F}_{S}$ is the Boson-Fock Space (see [6]). This means that a wave function $\psi \in \mathcal{S}$ is actually a sequence of functions $\psi=\left(\psi^{(n)}\right)_{n=0}^{\infty}$, where $\psi^{(n)}=$ $\psi^{(n)}\left(x, q_{1}, \ldots, q_{n}\right)$ (for $n \geq 1$ ) is invariant under permutation of $q_{1}, \ldots, q_{n}$ (clearly $\psi^{(0)}=\psi^{(0)}(x)$ ). $\mathcal{S}$ is a separable Hilbert-Space with the inner product given by

$$
\begin{aligned}
& \langle\phi, \psi\rangle_{\mathcal{S}}:=\int_{\mathbb{R}_{x}^{3}} \phi^{(0)}(x) \overline{\psi^{(0)}(x)} d x \\
& +\sum_{n=1}^{\infty} \int_{\mathbb{R}_{x}^{3}} \int_{\mathbb{R}_{q^{3 n}}^{3 n}} \phi^{(n)}\left(x, q^{(n)}\right) \\
& \overline{\psi^{(n)}\left(x, q^{(n)}\right)} d x d q^{(n)}
\end{aligned}
$$

where $q^{(n)}:=\left(q_{1}, \ldots, q_{n}\right)$ for $n \geq 1$.

The physical interpretation of $\mid \psi^{(n)}\left(x, q_{1}, \ldots\right.$, $\left.q_{n}\right)\left.\right|^{2}$ is the probability of finding the electron in an infinitesimal neighbourhood of $x$ (electron position space) and $n$ phonons in an infinitesimal neighbourhood of $q^{(n)}$ ( phonon momentum space). The electron position density is given by

$$
\begin{gathered}
n(x)=\left|\psi^{(0)}(x)\right|^{2}+\sum_{n=1}^{\infty} \int_{\mathbb{R}_{q^{(n)}}^{3 n}} \mid \\
\left.\psi^{(n)}\left(x, q^{(n)}\right)\right|^{2} d q^{(n)}
\end{gathered}
$$

and the current density by

$$
\begin{aligned}
J(x)= & \frac{\hbar}{m^{*}} \operatorname{Im}\left(\overline{\psi^{(0)}(x)} \nabla_{x} \psi^{(0)}(x)\right) \\
+ & \frac{\hbar}{m^{*}} \sum_{n=1}^{\infty} \int_{\mathbb{R}_{q^{3 n}}^{3 n}} \\
& \operatorname{Im}\left(\overline{\psi^{(n)}\left(x, q^{(n)}\right)} \nabla_{x} \psi^{(n)}\left(x, q^{(n)}\right)\right) d q^{(n)}
\end{aligned}
$$


where $\hbar$ is the Planck-constant and $m^{*}$ the electron mass.

The three terms of the Hamiltonian $H$ have the following form:

$$
H_{e}=-\frac{\hbar^{2}}{2 m^{*}} \Delta+V(x),
$$

$V(x)$ denoting a given real valued external potential. The Hamiltonian for the phonons is

$$
H_{p}=\hbar \int_{\mathbb{R}_{q}^{3}} a_{q}^{+} a_{q}\left(\omega(q)+Z\left(D_{q}\right)\right) d q
$$

with the annihilation and creation operators

$$
\begin{aligned}
& \left(a_{q} \phi\right)^{(n)}\left(x, q^{(n)}\right)=\sqrt{n+1} \phi^{(n+1)}\left(x, q, q^{(n)}\right) \\
& n=0,1, \ldots, \\
& \left(a_{q}^{+} \phi\right)^{(n)}\left(x, q^{(n)}\right) \\
& = \begin{cases}\frac{1}{\sqrt{n}} \sum_{l=1}^{n} \delta\left(q-q_{l}\right) \phi^{(n-1)}\left(x, l q^{(n)}\right) & n=1,2, \ldots \\
0 & n=0\end{cases}
\end{aligned}
$$

where we introduced the notation $l q^{(n)}:=\left(q_{1}, \ldots\right.$, $\left.q_{l-1}, q_{l+1}, \ldots, q_{n}\right)$.

In the phonon-Hamiltonian $\omega(q)$ is the real valued phonon-frequency and as a modification of the usual Hamiltonian we introduced the pseudodifferential operator $Z\left(D_{q}\right)$ which describes the phonon-phonon interactions. The mathematical reason for introducing $Z\left(D_{q}\right)$ is that we would like to have an orthonormal basis (ONB) of $\mathcal{F}_{s}$ consisting of eigenfunctions of $H_{p}$, which can be physically interpreted that the phonons are driven into states of thermal equilibrium. If we use the definition of the annihilation and creation operators then $H_{p}$ can be written as

$$
\begin{aligned}
& \left(H_{p} \psi\right)^{(n)}\left(x, q^{(n)}\right) \\
& =\hbar \sum_{l=1}^{n} \omega\left(q_{l}\right) \psi^{(n)}\left(x, q^{(n)}\right) \\
& \quad+\frac{\hbar}{(2 \pi)^{3}} \sum_{l=1}^{n} \int_{\mathbb{R}_{q}^{3}} \hat{Z}\left(q_{l}-q\right) \psi^{(n)}\left(x, q, l q^{(n)}\right) d q,
\end{aligned}
$$

where $\hat{Z}$ denotes the Fourier transform of the function $Z . \hat{Z}$ is supposed to be real valued. Here and in the sequel we set $\sum_{l=1}^{0} c_{l}:=0$. Finally the electronphonon interaction Hamiltonian is given by

$$
H_{e-p}=i \hbar \int_{\mathbb{R}_{q}^{3}} F(q)\left(a_{q} \mathrm{e}^{i q x}-a_{q}^{+} \mathrm{e}^{-i q x}\right) d q
$$

where the term with the annihilation operator models phonon absorption and the one with the creation operator models phonon emission. The real valued function $F(q)$ describes the details of the electron-phonon interaction. Again using the definitions of $a_{q}$ and $a_{q}^{+}$the interaction term reads

$$
\begin{aligned}
& \left(H_{e-p} \psi\right)^{(n)}\left(x, q^{(n)}\right) \\
& =i \hbar \sqrt{n+1} \int_{\mathbb{R}_{q}^{3}} F(q) \mathrm{e}^{i q x} \psi^{(n+1)}\left(x, q, q^{(n)}\right) d q \\
& -\frac{i \hbar}{\sqrt{n}} \sum_{l=1}^{n} F\left(q_{l}\right) \mathrm{e}^{-i q_{l} x} \psi^{(n-1)}\left(x, q^{(n)}\right) .
\end{aligned}
$$

Remark Since $V, \hat{Z}, F$ and $\omega$ are real-valued easy calculations show that the Hamiltonian $H$ is formally self-adjoint (for $H_{e-p}$ see also [6], p. 209f, Segal quantization). If we further assume that $F(p)=-F(-p)$ then the Fockspace Schrödinger equation is time reversible in the sense that the Hamiltonian $H$ commutes with the symmetry operation $q^{(n)} \rightarrow-q^{(n)}$ and conjugation.

To study the dynamics of the system we introduce the density operator $\rho: \mathcal{S} \rightarrow \mathcal{S}$ which fulfills the von-Neumann equation

$$
i \hbar \rho_{t}=[H, \rho],
$$

where $[A, B]:=A B-B A$ denotes the commutator of the operators $A$ and $B$. The operator $\rho$ is selfadjoint, positive and trace-class, therefore there exists an ONB $\left\{\rho_{l} \mid l \in \mathbb{N}\right\}$ of eigenfunctions of $\rho$ such that

$$
\rho \psi=\sum_{l=1}^{\infty} \mu_{l}\left\langle\psi, \rho_{l}\right\rangle_{\mathcal{S}} \rho_{l}
$$

where $\mu_{l} \geq 0$ are the corresponding eigenvalues. Using the eigenfunctions $\rho_{l}$ we introduce the 
density matrix elements

$$
\begin{aligned}
& r_{(n, m)}\left(x, q^{(n)} ; y, p^{(m)}, t\right) \\
& :=\sum_{l=1}^{\infty} \mu_{l} \rho_{l}^{(m)}\left(y, p^{(m)}, t\right) \overline{\rho_{l}^{(n)}\left(x, q^{(n)}, t\right)} n, m=0,1, \ldots
\end{aligned}
$$

which determine the density operator $\rho$ by

$$
\begin{aligned}
& (\rho \psi)^{(n)}\left(x, q^{(n)}, t\right) \\
& =\sum_{m=0}^{\infty} \int_{\mathbb{R}_{y}^{3}} \int_{\mathbb{R}_{p^{(m)}}^{3 m}} \psi^{(m)}\left(y, p^{(m)}, t\right) \\
& \frac{r_{(n, m)}\left(x, q^{(n)} ; y, p^{(m)}, t\right)}{d y d p^{(m)} .}
\end{aligned}
$$

It is easy to show that the eigenfunctions of $\rho$ fulfill the equation

$$
i \hbar \frac{\partial}{\partial t} \rho_{l}=H \rho_{l}
$$

which gives the connection between the vonNeumann dynamics and the Schrödinger picture (cf. [4]). Using (2.6) we obtain the equation

$$
\begin{aligned}
& i \hbar \frac{\partial}{\partial t} r_{(n, m)}\left(x, q^{(n)} ; y, q^{(m)}, t\right) \\
& =\sum_{l=1}^{\infty} \mu_{l}\left[\left(H \rho_{l}\right)^{(m)}\left(y, p^{(m)}, t\right) \overline{\rho_{l}^{(n)}\left(x, q^{(n)}, t\right)}\right. \\
& \left.\quad-\rho_{l}^{(m)}\left(y, p^{(m)}, t\right) \overline{\left(H \rho_{l}\right)^{(n)}\left(x, q^{(n)}, t\right)}\right]
\end{aligned}
$$

which describes the dynamics of the density matrix. As a first step to obtain a transport equation for the electrons we introduce

$$
\begin{aligned}
& W_{(n, m)}\left(x, v, q^{(n)} ; p^{(m)}, t\right) \\
& :=\frac{1}{(2 \pi)^{3}} \int_{\mathbb{R}_{\eta}^{3}} r_{(n, m)}\left(x+\frac{\hbar}{2 m^{*}} \eta, q^{(n)} ;\right. \\
& \left.\quad x-\frac{\hbar}{2 m^{*}} \eta, p^{(m)}, t\right) \mathrm{e}^{i v \cdot \eta} d \eta .
\end{aligned}
$$

The matrix $W:=\left(W_{(n, m)}\right)_{n, m=0,1, \ldots}$ is called Wigner Matrix. It is the Wigner-transformation of the density-matrix $r=\left(r_{(n, m)}\right)_{n, m=0,1, \ldots}$ with respect to the electron coordinates $x$ and $y$. Note that

$$
\begin{aligned}
& n(x, t) \\
& =\int_{\mathbb{R}_{v}^{3}} W_{(0,0)}(x, v, t) d v \\
& \quad+\sum_{n=1}^{\infty} \int_{\mathbb{R}_{v}^{3}} \int_{\mathbb{R}_{q^{(n)}}^{3 n}} W_{(n, n)}\left(x, v, q^{(n)} ; q^{(n)}, t\right) d q^{(n)} d v
\end{aligned}
$$

and

$$
\begin{aligned}
& J(x, t) \\
& =\int_{\mathbb{R}_{v}^{3}} v W_{(0,0)}(x, v, t) d v \\
& \quad+\sum_{n=1}^{\infty} \int_{\mathbb{R}_{v}^{3}} \int_{\mathbb{R}_{q^{(n)}}^{3 n}} v W_{(n, n)}\left(x, v, q^{(n)} ; q^{(n)}, t\right) d q^{(n)} d v .
\end{aligned}
$$

The transport equation satisfied by $W_{(n, m)}$ is easily derived from Eq. (2.7):

$$
\begin{aligned}
\frac{\partial}{\partial t} W_{(n, m)}+v & \cdot \nabla_{x} W_{(n, m)}+\theta_{\hbar}[V] W_{(n, m)} \\
& =Q_{p} W_{(n, m)}+\left(Q_{e-p} W\right)_{(n, m)}
\end{aligned}
$$

The operator

$$
\begin{aligned}
& \theta_{\hbar}[V] W_{(n, m)}\left(x, v, q^{(n)}, p^{(m)}, t\right) \\
&:=\frac{i}{(2 \pi)^{3}} \int \frac{V\left(x+\frac{\hbar}{2 m^{*}} \eta\right)-V\left(x-\frac{\hbar}{2 m^{*}} \eta\right)}{\hbar} \\
& \quad \cdot W_{(n, m)}\left(x, v^{\prime}, q^{(n)} ; p^{(m)}, t\right) \mathrm{e}^{i\left(v-v^{\prime}\right) \cdot \eta} d v^{\prime} d \eta
\end{aligned}
$$

is the usual pseudo-differential operator which stems from the external potential of the electron. The phonon-Hamiltonian gives the operator

$$
\begin{aligned}
& Q_{p} W_{(n, m)}\left(x, v, q^{(n)} ; p^{(m)}, t\right) \\
& :=-i\left(\sum_{k=1}^{m} \omega\left(p_{k}\right)-\sum_{k=1}^{n} \omega\left(q_{k}\right)\right) \\
& \times W_{(n, m)}\left(x, v, q^{(n)} ; p^{(m)}, t\right) \\
& -\frac{i}{(2 \pi)^{3}}\left[\sum_{k=1}^{m} \int_{\mathbb{R}_{p}^{3}} \hat{Z}\left(p_{k}-p\right)\right. \\
& \quad \times W_{(n, m)}\left(x, v, q^{(n)} ; p, k p^{(m)}, t\right) d p \\
& \quad-\sum_{k=1}^{n} \int_{\mathbb{R}_{q}^{3}} \hat{Z}\left(q_{k}-q\right) \\
& \left.\quad \times W_{(n, m)}\left(x, v, q_{, k} q^{(n)} ; p^{(m)}, t\right) d q\right]
\end{aligned}
$$


and finally we obtain for the electron-phonon interaction operator

$$
\begin{aligned}
& \left(Q_{e-p} W\right)_{(n, m)} \\
& \quad:=Q_{1}^{-} W_{(n, m+1)}+Q_{2}^{-} W_{(n+1, m)} \\
& \quad-Q_{1}^{+} W_{(n, m-1)}-Q_{2}^{+} W_{(n-1, m)}
\end{aligned}
$$

with

$$
\begin{aligned}
& Q_{1}^{-} W_{(n, m+1)} \\
& =\sqrt{m+1} \int_{\mathbb{R}_{p}^{3}} F(p) \mathrm{e}^{i p \cdot x} \\
& \times W_{(n, m+1)}\left(x, v-\frac{h}{2 m^{*}} p, q^{(n)} ; p, p^{(m)}, t\right) d p, \\
& Q_{2}^{-} W_{(n+1, m)}=\sqrt{n+1} \int_{\mathbb{R}_{q}^{3}} F(q) \mathrm{e}^{-i q \cdot x} \\
& \times W_{(n+1, m)}\left(x, v-\frac{h}{2 m^{*}} q, q, q^{(n)} ; p^{(m)}, t\right) d q, \\
& Q_{1}^{+} W_{(n, m-1)}=\frac{1}{\sqrt{m}} \sum_{k=1}^{m} F\left(p_{k}\right) \mathrm{e}^{-i p_{k} \cdot x} \\
& \times W_{(n, m-1)}\left(x, v+\frac{h}{2 m^{*}} p_{k}, q^{(n)} ; k p^{(m)}, t\right), \\
& Q_{2}^{+} W_{(n+1, m)}=\frac{1}{\sqrt{n}} \sum_{k=1}^{n} F(q k) \mathrm{e}^{i q_{k} \cdot x} \\
& \times W_{(n-1, m)}\left(x, v+\frac{h}{2 m^{*}} q_{k, k} q^{(n)} ; p^{(m)}, t\right) .
\end{aligned}
$$

We now introduce the phonon trace of the (Wigner) matrix $W$

$$
\begin{aligned}
w(x, v, t) & :=\left(\operatorname{tr}_{p} W\right)(x, v, t) \\
:= & W_{(0,0)}(x, v, t) \\
& +\sum_{n=1}^{\infty} \int_{\mathbb{R}_{q^{(n)}}^{3 n}} W_{(n, n)}\left(x, v, q^{(n)} ; q^{(n)}, t\right) d q^{(n)},
\end{aligned}
$$

which acts as the quantum-equivalent of the phase space distribution function of the electron in classical mechanics. Taking the phonon trace of (2.8) leads (after some calculations) to the transport equation

$$
\begin{aligned}
w_{t} & +v \cdot \nabla_{x} w+\theta_{\hbar}[V] w \\
& =2 \sum_{n=0}^{\infty} \sqrt{n+1} \int_{\mathbb{R}_{q}^{3}} \int_{\mathbb{R}_{q^{(n)}}^{3 n}} F(q)
\end{aligned}
$$

$$
\begin{aligned}
\cdot \operatorname{Re} e & {\left[\mathrm { e } ^ { i q \cdot x } \left(W_{(n, n+1)}\left(x, v-\frac{h}{2 m^{*}} q, q^{(n)} ; q, q^{(n)}, t\right)\right.\right.} \\
& \left.\left.-W_{(n, n+1)}\left(x, v+\frac{h}{2 m^{*}} q, q^{(n)} ; q, q^{(n)}, t\right)\right)\right] d q^{(n)} d q
\end{aligned}
$$

Remarks

- Note that $\operatorname{tr}_{p}\left(Q_{p} W\right)=0$.

- In the right hand side of (2.11) the subdiagonal elements $W_{(n, n+1)}$ are still present, which means that we do not have a closed equation for $w(x, v, t)$.

- Note that $n(x, t)=\int_{\mathbb{R}_{v}^{3}} w(x, v, t) d v$ and $J(x, t)=$ $\int_{\mathbb{R}_{v}^{3}} v w(x, v, t) d v$.

\section{WEAK ELECTRON-PHONON INTERACTION}

To derive an approximating closed equation for $w(x, v, t)$ we now assume that the electron-phonon interaction is small. Therefore we write in (2.5) $\varepsilon F(q)$ instead of $F(q)$ with $0<\varepsilon \ll 1$ and treat the problem with methods of asymptotic analysis for $\varepsilon \rightarrow 0$. The now $\varepsilon$-dependent Wigner matrix is solution of the transport equation

$$
L W^{\varepsilon}=Q_{p} W^{\varepsilon}+\varepsilon Q_{e-p} W^{\varepsilon}
$$

where we introduced the Wigner transport operator

$$
L:=\frac{\partial}{\partial t}+v \cdot \nabla_{x}+\theta_{\hbar}[V]
$$

$Q_{p}$ and $Q_{e-p}$ are now considered to act on the Wigner matrix $W^{\varepsilon}$ as defined in (2.8). For $W^{\varepsilon}$ we make the ansatz

$$
W^{\varepsilon}:=W^{0}+\varepsilon W^{1}+\varepsilon^{2} W^{2}+\varepsilon^{3} W^{3}+O\left(\varepsilon^{4}\right) .
$$

For the initial condition we assume

$$
W^{\varepsilon}(t=0)=w_{I}^{0} A
$$


where $w_{I}^{0}=w^{0}(t=0)$ is a given (Wigner) function of $x$ and $v$ and $A$ is defined as the density matrix corresponding to the operator ( $c f$. [1])

$$
T:=\frac{1}{\operatorname{tr}_{p}\left(\mathrm{e}^{\left.-\beta H_{p}^{0}\right)}\right.} \mathrm{e}^{-\beta H_{p}^{0}}, \quad \text { with } \quad H_{p}=\mathrm{Id}_{x} \otimes H_{p}^{0}
$$

( $H_{p}^{0}$ is the phonon operator acting on $\mathcal{F}_{S}$ ). The exact definition of $A$ will be given after we have introduced a special ONB in Lemma 3.1. The operator $T$ describes the phonons in a state of thermal equilibrium, where $\beta$ is a constant (indirectly proportional to the lattice temperature). Note that $T$ is normalized such that $\operatorname{tr}_{p} T=1$.

We make the following assumption on $H_{p}^{0}$ :

(A1) $\omega(q)$ and $\hat{Z}(q)$ are such that there exists an ONB of real valued eigen-functions $\left\{\psi_{k}(q) \mid\right.$ $k \in \mathbb{N}\}$ in $L^{2}\left(\mathbb{R}_{q}^{3}\right)$ and eigenvalues $\lambda_{k} \in \mathbb{R}$ such that

$$
\begin{aligned}
& \hbar \omega(q) \psi_{k}(q)+\frac{\hbar}{(2 \pi)^{3}} \int_{\mathbb{R}_{q^{\prime}}^{3}} \hat{Z}\left(q-q^{\prime}\right) \psi_{k}\left(q^{\prime}\right) d q^{\prime} \\
& =\lambda_{k} \psi_{k}(q) .
\end{aligned}
$$

Note that (A1) holds if growth conditions on $\omega(q)$ and on $Z=Z(x)$ at $x=q=\infty$ are imposed (confinement of phonons). The eigenfunctions can be chosen real valued because $\omega(q)$ and $\hat{Z}$ are real valued. With this assumption we have the following

LeMma 3.1 If (A1) holds, then there exists an $O N B$ of $\mathcal{F}_{S}$ consisting of eigenfunctions of $H_{p}^{0}$.

Proof Define

$$
\begin{aligned}
& \psi_{\vec{k}}^{(n)}\left(q_{1}, \ldots, q_{n}\right) \\
& \quad:=\frac{1}{\sqrt{n !}} \sum_{\sigma \in P^{n}} \psi_{k_{\sigma(1)}}\left(q_{1}\right) \cdot \ldots \cdot \psi_{k_{\sigma(n)}}\left(q_{n}\right)
\end{aligned}
$$

where $\vec{k}=\left(k_{1}, \ldots, k_{n}\right)$ is a multiindex and $P^{n}$ is the permutation group of $n$ elements. Because of the structure of $H_{p}$ it is obvious that

$$
\begin{aligned}
H_{p}^{0} \psi_{\vec{k}}^{(n)}\left(q^{(n)}\right) & =\lambda_{\vec{k}} \psi_{\vec{k}}^{(n)}\left(q^{(n)}\right) \quad \text { with } \\
\lambda_{\vec{k}} & :=\sum_{j=1}^{n} \lambda_{k_{j}},
\end{aligned}
$$

$\lambda_{k_{j}}$ being the eigenvalue corresponding to $\psi_{k_{j}}$. By definition the functions $\psi_{\vec{k}}\left(q_{1}, \ldots, q_{n}\right)$ are invariant under permutation of the arguments and therefore $\psi_{\vec{k}}:=\left(0, \ldots, 0, \psi_{\vec{k}}^{(n)}\left(q^{(n)}\right), 0, \ldots\right) \in \mathcal{F}_{S}$. Adding the vacuum state $\psi^{0}:=(1,0,0, \ldots)$ we finally find that the so constructed set $\left\{\psi_{\vec{k}}\right\}$ is an ONB of $\mathcal{F}_{S}$ because $\left(\psi_{k}\right)_{k \in \mathbb{N}}$ is an ONB of $L^{2}\left(\mathbb{R}_{q}^{3}\right), c f$. [5].

Now we use the ONB constructed above to represent the matrix $A$ of the initial condition (3.3). To obtain a unique representation we use only multiindices $\vec{k}=\left(k_{1}, \ldots, k_{n}\right)$ ordered such that $k_{1} \leq \cdots \leq k_{n}$. We thus obtain $\forall \phi \in \mathcal{F}_{S}$ :

$$
\begin{aligned}
& \left(H_{p}^{0} \phi\right)^{(n)}\left(q^{(n)}\right) \\
& =\sum_{\substack{\vec{k} \in \mathbb{N}^{n} \\
k_{1} \leq \cdots \leq k_{n}}} \lambda_{\vec{k}} \int_{\substack{\mathbb{R}_{p}^{3 n} \\
p^{(n)}}} \phi^{(n)}\left(p^{(n)}\right) \psi_{\vec{k}}^{(n)}\left(p^{(n)}\right) d p^{(n)} \psi_{\vec{k}}^{(n)}\left(q^{(n)}\right) .
\end{aligned}
$$

Using the definition of the operator $T$ we can write

$$
\begin{aligned}
& (T \phi)^{(n)}\left(q^{(n)}\right) \\
& \quad=\sum_{m=1}^{\infty} \int_{\substack{\mathbb{R}_{p}^{3 m} \\
p^{(m)}}} \phi^{(m)}\left(p^{(m)}\right) A_{(n, m)}\left(q^{(n)}, p^{(m)}\right) d p^{(m)}
\end{aligned}
$$

where we have

$$
\begin{aligned}
& A_{(n, n)}\left(q^{(n)}, p^{(n)}\right) \\
& =\frac{1}{\operatorname{Tr}} \sum_{\substack{\vec{k} \leq \mathbb{N}^{n} \\
k_{1} \leq \cdots \leq k_{n}}} \mathrm{e}^{-\beta \lambda_{\vec{k}}} \psi_{\vec{k}}^{(n)}\left(p^{(n)}\right) \psi_{\vec{k}}^{(n)}\left(q^{(n)}\right) \\
& \quad n=1,2, \ldots
\end{aligned}
$$

with $\operatorname{Tr}:=\sum_{n} \sum_{\substack{\vec{k} \in \mathbb{N}^{n} \\ k_{1} \leq \cdots \leq k_{n}}} \mathrm{e}^{-\beta \lambda_{\vec{k}}}$ and because of the construction of the ONB we have

$$
A_{(n, m)}\left(q^{(n)}, p^{(m)}\right)=0 \quad n \neq m .
$$

Plugging the ansatz (3.2) into Eq. (3.1) we obtain by equating the coefficients of equal powers of $\varepsilon$ :

$$
\begin{aligned}
& L W^{0}-Q_{p} W^{0}=0 \\
& L W^{1}-Q_{p} W^{1}=Q_{e-p} W^{0} \\
& L W^{2}-Q_{p} W^{2}=Q_{e-p} W^{1} \\
& L W^{3}-Q_{p} W^{3}=Q_{e-p} W^{2}
\end{aligned}
$$


and from (3.3) we obtain the initial conditions

$$
\begin{aligned}
& W^{0}(t=0)=w^{0} A \\
& W^{1}(t=0)=W^{2}(t=0)=W^{3}(t=0)=0 .
\end{aligned}
$$

Using the first equation of (3.4) and the initial condition for $W^{0}$ the separation ansatz $W_{n, m}^{0}=$ $w^{0}(x, v, t) M_{n, m}\left(q^{n}, p^{m}\right)$ shows that $M=A$, i.e., $W^{0}=w^{0} A$. A short calculation gives $Q_{p} A=0$, therefore also $Q_{p} W^{0}=0$.

In the following we will use the convention that for a superscript $\alpha \in\{0,1,2,3, \varepsilon\}$ the function $w^{\alpha}$ will denote the phonon trace of the matrix $W^{\alpha}$, i.e., $w^{\alpha}:=\operatorname{tr}_{p} W^{\alpha}$.

\section{Remarks}

- Note that this notation is consistent for $W^{0}$ because of the normalization of $A, \operatorname{tr}_{p} A=1$.

- The interpretation of the structure of $W^{0}=w^{0} A$ is that if there is no electron-phonon interaction at all (i.e., $\varepsilon=0$ ) the phonons will be in a state of thermal equilibrium which is given by the operator $T$.

If we take the phonon traces of Eqs. (3.4) we are thus led to the equations

$$
\begin{aligned}
& L w^{0}=0 \\
& L w^{1}=\operatorname{tr}_{p}\left(Q_{e-p} W^{0}\right) \\
& L w^{2}=\operatorname{tr}_{p}\left(Q_{e-p} W^{1}\right) \\
& L w^{3}=\operatorname{tr}_{p}\left(Q_{e-p} W^{2}\right) .
\end{aligned}
$$

We have already used $\operatorname{tr}_{p}\left(Q_{p} W^{\alpha}\right)=0$ and another calculation shows that $\operatorname{tr}_{p}\left(Q_{e-p} W^{0}\right)=0$. Actually this can be seen easily by taking into account the fact that $A_{(n, m)}=0$ for $n \neq m$ and therefore $\left(Q_{e-p} W^{0}\right)_{(n, n)}=0 . \forall n \geq 0$. With a similar argument one can see that $\operatorname{tr}_{p}\left(Q_{e-p} W^{2}\right)=0$. So taking into account the initial conditions (3.5) we have found $w^{1} \equiv w^{3} \equiv 0$ and, formally,

$$
L w^{\varepsilon}=\varepsilon^{2} \operatorname{tr}_{p}\left(Q_{e-p} W^{1}\right)+O\left(\varepsilon^{4}\right)
$$

where we have of course $w^{\varepsilon}=\operatorname{tr}_{p} W^{\varepsilon}=w^{0}+$ $\varepsilon^{2} w^{2}+O\left(\varepsilon^{4}\right)$.

The case $V \equiv$ const.
$W^{1}$ is now calculated from

$$
L W^{1}-Q_{p} W^{1}=Q_{e-p}\left(w^{\varepsilon} A\right)
$$

which is the second equation of (3.4) with $w^{0}$ replaced by $w^{\varepsilon}$. If we are able to solve Eq. (3.7) for $W^{1}$ we will have a closed equation for $w^{\varepsilon}$ which is exact up to the order $\varepsilon^{4}$. To do so we assume now $V \equiv$ const. which means $\theta_{\hbar}[V] \equiv 0$. In this simple case Eq. (3.7) becomes

$$
\frac{\partial}{\partial t} W^{1}+v \cdot \nabla_{x} W^{1}-Q_{p} W^{1}=Q_{e-p}\left(w^{\varepsilon} A\right)
$$

which can be solved explicitely by means of a separation ansatz and the variation of the constants formula. Using the orthogonality properties of the eigenfunctions of $H_{p}^{0}$ one finally obtains after long calculations

$$
\begin{aligned}
& \operatorname{tr}_{p}\left(Q_{e-p} W^{1}\right) \\
& =\frac{2}{\operatorname{Tr}} \int_{\tau=0}^{t} \int_{\mathbb{R}_{p}^{3}} \int_{\mathbb{R}_{q}^{3}} \sum_{n=0}^{\infty} \sum_{\substack{\vec{k} \in \mathbb{N}^{n+1} \\
k_{1} \leq \cdots \leq k_{n+1}}} \\
& \sum_{j=1}^{n+1} \operatorname{Re}\left\{\mathrm{e}^{i(q-p) \cdot x+i p \cdot v \tau} \cdot F_{k_{j}}(p) F_{k_{j}}(q) \mathrm{e}^{\frac{i}{\hbar} \lambda_{k_{j}} \tau}\right. \\
& \left.\left(\mathrm{e}^{-\beta \lambda_{\vec{k}}} D_{1}^{0}-\mathrm{e}^{-\beta \lambda_{j} \vec{k}} D_{2}^{0}\right)\right\} d q d p d \tau
\end{aligned}
$$

with $F_{k_{j}}(p):=F(p) \psi_{k_{j}}(p)$ and

$$
\begin{gathered}
D_{1}^{0}:=\mathrm{e}^{-i \frac{\hbar}{2 m^{*}} p \cdot q \tau} w^{\varepsilon}\left(x-\left(v-\frac{\hbar}{2 m^{*}} q\right) \tau,\right. \\
\left.v-\frac{\hbar}{2 m^{*}}(p+q), t-\tau\right) \\
-\mathrm{e}^{i \frac{\hbar}{2 m^{*}} p \cdot q \tau} w^{\varepsilon}\left(x-\left(v+\frac{\hbar}{2 m^{*}} q\right) \tau,\right. \\
\left.v-\frac{\hbar}{2 m^{*}}(p-q), t-\tau\right) \\
D_{2}^{0}:=\mathrm{e}^{-i \frac{\hbar}{2 m^{*}} p \cdot q \tau} w^{\varepsilon}\left(x-\left(v-\frac{\hbar}{2 m^{*}} q\right) \tau,\right. \\
\left.v+\frac{\hbar}{2 m^{*}}(p-q), t-\tau\right) \\
-\mathrm{e}^{i \frac{\hbar}{2 m^{*}} p \cdot q \tau} w^{\varepsilon}\left(x-\left(v+\frac{\hbar}{2 m^{*}} q\right) \tau,\right. \\
\left.v+\frac{\hbar}{2 m^{*}}(p+q), t-\tau\right) .
\end{gathered}
$$


So in the case of $V \equiv$ const. we obtain the transport equation for $w^{\varepsilon}$

$$
\frac{\partial}{\partial t} w^{\varepsilon}+v \cdot \nabla_{x} w^{\varepsilon}=\varepsilon^{2} I_{\text {scat }}^{0}+O\left(\varepsilon^{4}\right)
$$

where $I_{\text {scat }}^{0}$ is the term on the right hand side of (3.8).

\section{Remarks}

- The most important property of $I_{\text {scat }}^{0}$ is the nonlocality in time. This expresses the fact that the scattering term has a memory of the whole history of the states of the system, i.e., phonon scattering is nonlocal in time when a fully quantum viewpoint is taken.

- An easy calculation shows that if $F_{j}(q)$ is either symmetric or antisymmetric, i.e., $F_{j}(q)=$ $F_{j}(-q)$ or $F_{j}(q)=-F_{j}(-q)$, then Eq. (3.9) (without the $O\left(\varepsilon^{4}\right)$-term) is time reversible (i.e., the equation is invariant under the transformation $t \rightarrow-t, v \rightarrow-v) . F_{j}$ has such symmetry properties, for example, if $F$ is antisymmetric and $\psi_{j}$ is symmetric or antisymmetric which is the case if $\omega$ and $\hat{Z}$ are symmetric.

The case of a constant electric field:

We now consider a linear potential $V=-E \cdot x$ where $E$ is the constant electric field. In this case the pseudo-differential operator $\theta_{\hbar}[V]$ becomes the differential operator $-\left(1 / m^{*}\right) E \cdot \nabla_{\nu}$ which means that the operator $L$ is the Vlasov transport operator

$$
L=\frac{\partial}{\partial t}+v \cdot \nabla_{x}-B \cdot \nabla_{v}, B=\frac{E}{m^{*}} .
$$

So we can again solve Eq. (3.7) by the method of characteristics and by similar calculations as in the case of $V \equiv$ const. we derive the transport equation

$$
\frac{\partial}{\partial t} w^{\varepsilon}+v \cdot \nabla_{x} w^{\varepsilon}-B \cdot \nabla_{v} w^{\varepsilon}=\varepsilon^{2} I_{\text {scat }}^{B}+O\left(\varepsilon^{4}\right)
$$

where we have to replace $D_{1}^{0}$ and $D_{2}^{0}$ in the expression (3.8) by

$$
\begin{aligned}
D_{1}^{B}:= & \mathrm{e}^{i p \frac{B}{2} \tau^{2}-i \frac{\hbar}{2 m^{*}} p \cdot q \tau} w^{\varepsilon} \\
& \left(x-\left(v-\frac{\hbar}{2 m^{*}} q\right) \tau-\frac{B}{2} \tau^{2},\right. \\
& \left.v-\frac{\hbar}{2 m^{*}}(p+q)+B \tau, t-\tau\right) \\
& -\mathrm{e}^{i p \frac{B}{2} \tau^{2}+i \frac{\hbar}{2 m^{*}} p \cdot q \tau} w^{\varepsilon} \\
& \left(x-\left(v+\frac{\hbar}{2 m^{*}} q\right) \tau-\frac{B}{2} \tau^{2},\right. \\
& \left.v-\frac{\hbar}{2 m^{*}}(p-q)+B \tau, t-\tau\right), \\
D_{2}^{B}:= & \mathrm{e}^{i p \frac{B}{2} \tau^{2}-i \frac{\hbar}{2 m^{*}} p \cdot q \tau} w^{\varepsilon} \\
& \left(x-\left(v-\frac{\hbar}{2 m^{*}} q\right) \tau-\frac{B}{2} \tau^{2},\right. \\
& \left.v+\frac{\hbar}{2 m^{*}}(p-q)+B \tau, t-\tau\right) \\
& -\mathrm{e}^{i p \frac{B^{2}}{2}+\frac{\hbar}{2 m^{*}} p \cdot q \tau} w^{\varepsilon} \\
& \left(x-\left(v+\frac{\hbar}{2 m^{*}} q\right) \tau-\frac{B}{2} \tau^{2},\right. \\
& \left.v+\frac{\hbar}{2 m^{*}}(p+q)+B \tau, t-\tau\right) .
\end{aligned}
$$

We can write the scattering term also in pseudodifferential operator (PDO) form. For this purpose we introduce

$$
\begin{aligned}
f(x, k) & :=\int_{\mathbb{R}_{q}^{3}} \mathrm{e}^{-i q x} F(q) \psi_{k}(q) d q, \\
\delta f(x, \eta, k) & :=f(x+\eta, k)-f(x-\eta, k), \\
\mu f(x, \eta, k) & :=f(x+\eta, k)+f(x-\eta, k) .
\end{aligned}
$$

Using this notation we obtain

$$
\begin{aligned}
& I_{\text {scat }}^{B}=\frac{1}{\operatorname{Tr}} \int_{\tau=0}^{t} \sum_{n=0}^{\infty} \sum_{\substack{k \in N^{n+1} \\
k_{1} \leq \cdots \leq k_{n+1}}} \\
& \sum_{j=1}^{n+1} \operatorname{Re}\left\{\mathrm{e}^{\frac{i}{\hbar \lambda_{j} \tau} \delta f\left(x, \frac{\hbar}{2 m^{*} i} \nabla_{v}, k_{j}\right)}\right. \\
& \left(\left(\mathrm{e}^{-\beta \lambda_{\vec{k}}}+\mathrm{e}^{-\beta \lambda_{j} \vec{k}}\right) \delta f\right.
\end{aligned}
$$




$$
\begin{aligned}
& \left(x-\frac{B}{2} \tau^{2}-v \tau, \frac{\hbar}{2 m^{*} i} \nabla_{v, 2}, k_{j}\right) \\
& +\left(\mathrm{e}^{-\beta \lambda_{\vec{k}}}-\mathrm{e}^{-\beta \lambda_{j^{k}}}\right) \mu f \\
& \left.\left(x-\frac{B}{2} \tau^{2}-v \tau, \frac{\hbar}{2 m^{*} i} \nabla_{v, 2}, k_{j}\right)\right) \\
& \left.w^{\varepsilon}\left(x-\frac{B}{2} \tau^{2}-v \tau, v+B \tau, t-\tau\right)\right\} d \tau
\end{aligned}
$$

where the notation $\nabla_{v, 2}$ signifies that the PDO acts only on the second argument of $w$.

\section{SCALING LIMITS}

We shall assume in this chapter that $\operatorname{Tr}=1$. For the independent variables we introduce the scaling

$$
t=\nu \tilde{t}, \quad x=\gamma_{x} \tilde{x}, \quad v=\gamma_{v} \tilde{v}, \quad q=\alpha \tilde{q}
$$

and for the other occuring quantities we have

$$
\begin{gathered}
w^{\varepsilon}(x, v, t)=\tilde{w}^{\varepsilon}(\tilde{x}, \tilde{v}, \tilde{t}), \quad \psi_{k}(q)=\alpha^{-\frac{3}{2}} \tilde{\psi}_{k}(\tilde{q}), \\
E=A_{E} \tilde{E}, \quad F(q)=A_{F} \tilde{F}(\tilde{q}), \quad \lambda_{\vec{k}}=A_{P} \tilde{\lambda}_{\vec{k}} .
\end{gathered}
$$

The scaling is chosen such that the set $\left\{\tilde{\psi}_{k}\right\}$ is an ONB of $L^{2}\left(\mathbb{R}_{\tilde{q}}^{3}\right)$. Note that

$$
\begin{aligned}
{\left[\psi^{(n)}\right] } & =\left(m^{3}\right)^{\frac{n-1}{2}},[q]=m^{-1}, \\
{[E] } & =k g m s^{-2},[F]=m^{3 / 2} s^{-1} .
\end{aligned}
$$

We introduce the 3 dimensionless parameters

$$
\varepsilon:=\gamma_{v} m^{*} \frac{A_{F}}{A_{E}} \alpha^{\frac{3}{2}}, \sigma:=\frac{m^{*} \gamma_{v}}{\nu A_{E}}, \kappa:=\alpha \gamma_{x} .
$$

Setting $\nu=(\gamma x / \gamma v), \gamma_{x}=\left(\hbar / m^{*} \gamma_{v}\right)$ and $\gamma_{v}=\left(\hbar A_{E} /\right.$ $\left.m^{*} A_{P}\right)$ we obtain the scaled equation

$$
\begin{aligned}
& \sigma w_{t}+ \sigma v \cdot \nabla_{x} w-E \cdot \nabla_{v} w \\
&= \varepsilon^{2} \sum_{n=0}^{\infty} \sum_{\substack{\vec{k} \in \mathbb{N}^{n+1} \\
k_{1} \leq \cdots \leq k_{n+1}}} \sum_{j=1}^{n+1} \int_{\tau=0}^{\frac{t}{\sigma}} \\
& \operatorname{Re}\left\{\mathrm{e}^{i \sigma \lambda_{k_{j}} \tau f\left(\kappa x, \frac{\kappa}{2 i} \nabla_{\nu}, k_{j}\right)}\right. \\
&\left(\left(\mathrm{e}^{-\beta \lambda_{\vec{k}}}+\mathrm{e}^{-\beta \lambda_{j} \vec{k}}\right)\right.
\end{aligned}
$$

$$
\begin{gathered}
\delta f\left(\kappa x-\sigma \frac{E}{2} \tau^{2}-\sigma v \tau,\right. \\
\left.\frac{\kappa}{2 i} \nabla_{v, 2}, k_{j}\right) \\
+\left(\mathrm{e}^{-\beta \lambda_{\vec{k}}}-\mathrm{e}^{-\beta \lambda_{j} \vec{k}}\right) \\
\mu f\left(\kappa x-\sigma \frac{E}{2} \tau^{2}-\sigma v \tau,\right. \\
\left.\left.\frac{\kappa}{2 i} \nabla_{v, 2}, k_{j}\right)\right) \\
w^{\varepsilon}\left(x-\sigma \frac{E}{2} \tau^{2}-\sigma v \tau,\right. \\
v+E \tau, t-\sigma \tau)\} d \tau
\end{gathered}
$$

where we have dropped " " for the scaled quantities. The scaling is chosen such that $\sigma$ is indirectly proportional to the strength of the electric field, $\kappa$ is proportional to the scaling parameter of $q$ and $\varepsilon$ is proportional to the strength of the electron-phonon interaction.

\section{Limit 1}

Taking the limit $\sigma \rightarrow 0$ (which means we consider strong electric fields) we formally obtain the limiting equation

$$
\begin{aligned}
w_{t}+v \cdot \nabla_{x} w- & \frac{1}{\sigma} E \cdot \nabla_{v} w \\
= & \frac{\varepsilon^{2}}{\sigma} \sum_{n=0}^{\infty} \sum_{\substack{\vec{k} \in N^{n+1} \\
k_{1} \leq \cdots \leq k_{n+1}}} \\
& \sum_{j=1}^{n+1} \int_{\tau=0}^{\infty}\left(\mathrm{e}^{-\beta \lambda_{\vec{k}}}+\mathrm{e}^{-\beta \lambda_{\vec{k}}}\right) \\
& \delta f\left(\kappa x, \frac{\kappa}{2 i} \nabla_{v}, k_{j}\right) \\
& \delta f\left(\kappa x, \frac{\kappa}{2 i} \nabla_{v}, k_{j}\right) \\
& w^{\varepsilon}(x, v+E \tau, t) d \tau .
\end{aligned}
$$

\section{Remarks}

- Here we assumed that $F_{k_{j}}(q)=F(q) \psi_{k_{j}}(q)$ is symmetric or antisymmetric. Then it follows 
that the operator $\overline{\delta f} \delta f$ maps real functions into real ones and the operator $\overline{\delta f} \mu f$ maps real functions into purely imaginary ones.

- Note that $\varepsilon=A_{F} \sigma \nu \alpha^{(3 / 2)}$. Assuming that $\nu$ and $\alpha$ are constant, $\sigma \rightarrow 0$ obviously implies $\varepsilon \rightarrow 0$ and thus $\left(\varepsilon^{2} / \sigma\right) \rightarrow 0$.

If we then take the limit $\kappa \rightarrow 0$ (small wave vectors $q$ ) the PDOs become differential operators

$$
\begin{aligned}
& \delta f\left(\kappa x, \frac{\kappa}{2 i} \nabla_{v}, k_{j}\right) w(x, v, t) \\
& \quad=i \kappa \nabla_{x} f\left(0, k_{j}\right) \cdot \nabla_{v} w(x, v, t)+O\left(\kappa^{3}\right)
\end{aligned}
$$

and we derive

$$
\begin{aligned}
w_{t}+v & \cdot \nabla_{x} w-\frac{1}{\sigma} E \cdot \nabla_{v} w \\
& =\frac{\varepsilon^{2} \kappa^{2}}{\sigma} \int_{\tau=0}^{\infty} \nabla_{v}^{T} M \nabla_{v} w(x, v+E \tau, t) d \tau
\end{aligned}
$$

with the matrix

$$
\begin{aligned}
& M= \sum_{n=0}^{\infty} \sum_{\substack{\vec{k} \in \mathbb{N}^{n+1} \\
k_{1} \leq \cdots \leq k_{n+1}}}{ }^{n+1}\left(\mathrm{e}^{-\beta \lambda_{\vec{k}}}+\mathrm{e}^{-\beta \lambda_{j^{\vec{k}}}}\right) G_{k_{j}} \otimes G_{k_{j}}, \\
& G_{k_{j}=1}=\int_{\mathbb{R}_{q}^{3}} F(q) \psi_{k_{j}}(q) q d q .
\end{aligned}
$$

Using the equality

$$
\begin{gathered}
\int_{\tau=0}^{\infty} \int_{\mathbb{R}_{\xi}} h(\xi+\tau) h(\xi) d \xi d \tau \\
=\frac{1}{2}\left(\int_{\mathbb{R}_{\xi}} h(\xi) d \xi\right)^{2}
\end{gathered}
$$

we can proof easily that the scattering term in (4.3) is dissipative.

\section{Limit 2}

We obtain another simplified scattering term if we are only interested in the equation for small times.
We set $\sigma=1$ (electric field strength of order one) and take first the limit $\kappa \rightarrow 0$ which leads to the approximation

$$
\begin{aligned}
& w_{t}+v \cdot \nabla_{x} w-E \cdot \nabla_{v} w \\
& =\varepsilon^{2} \kappa^{2} \int_{\tau=0}^{t} \cos \left(\lambda_{k_{j}} \tau\right) \nabla_{v}^{T} \\
& \quad M \nabla_{v, 2} w\left(x+\frac{E}{2} \tau^{2}-v \tau, \quad v+E \tau, t-\tau\right) d \tau .
\end{aligned}
$$

Taylor expansion with respect to $t$ gives the equation

$$
\begin{aligned}
w_{t}+v & \cdot \nabla_{x} w-E \cdot \nabla_{v} w \\
& =\varepsilon^{2} \kappa^{2} t \nabla_{v}^{T} M \nabla_{v} w(x, v, t)+o\left(\varepsilon^{2} \kappa^{2} t\right) .
\end{aligned}
$$

Taking only the leading term of this expansion gives a dissipative (Fokker-Planck) scattering term.

\section{BARKER-FERRY EQUATION}

In the physical literature the Barker-Ferry equation is quite well known (see e.g. [2]). It is a transport equation for an electron in a constant electric field with a scattering term describing the electron-phonon interaction for the space homogenous case. To derive this equation we make the assumption that the function $F$ of the electronphonon interaction Hamiltonian (2.5) is a random variable $F=F(q, \alpha)$ (where $\alpha$ varies in an appropriate measure space) such that

$$
\langle F(p) F(q)\rangle=R(q) \delta(p-q)
$$

where $R(q)$ is a real valued function and $\langle\cdot\rangle$ denotes the average with respect to $\alpha$. This means that the random variables $F(p)$ and $F(q)$ are uncorrelated for $p \neq q$.

Repeating the asymptotic expansion of chapter 3 we note that the effect of the random interaction Hamiltonian is of order $\varepsilon$. Therefore it is reasonable to assume that $w^{0}=w^{0}(x, v, t)$ does not depend on $\alpha$. If we define $\bar{w}^{\varepsilon}:=\left\langle w^{\varepsilon}\right\rangle$ then taking 
the average of (3.10) and using the relation (5.1) leads to

$$
\begin{aligned}
& L \bar{w}^{\varepsilon}=\frac{2 \varepsilon^{2}}{\operatorname{Tr}} \int_{\tau=0}^{t} \int_{\mathbb{R}_{q}^{3}} \sum_{n=0}^{\infty} \sum_{\substack{\vec{k} \in \mathbb{N}^{n+1} \\
k_{1} \leq \cdots \leq k_{n+1}}} \\
& \sum_{j=1}^{n+1} \operatorname{Re}\left\{\mathrm{e}^{i q \cdot\left(v+\frac{B}{2} \tau\right) \tau} \cdot R(q) \Psi_{k_{j}}(q)^{2} \mathrm{e}^{\frac{i}{\hbar} \lambda_{k} \tau}\right. \\
& \left.\left(\mathrm{e}^{-\beta \lambda_{\vec{k}}} \bar{D}_{1}^{B}-\mathrm{e}^{-\beta \lambda_{j} \vec{k}} \bar{D}_{2}^{B}\right)\right\} \\
& d q d p d \tau+O\left(\varepsilon^{4}\right)
\end{aligned}
$$

with

$$
\begin{array}{r}
\bar{D}_{1}^{B}:=\mathrm{e}^{-i \frac{\hbar}{2 m^{*}} q^{2} \tau} \bar{w}^{\varepsilon}\left(x-\left(v-\frac{\hbar}{2 m^{*}} q\right) \tau-\frac{B}{2} \tau^{2},\right. \\
\left.v-\frac{\hbar}{m^{*}} q+B \tau, t-\tau\right) \\
-\mathrm{e}^{i \frac{\hbar}{2 m^{*}} q^{2} \tau} \bar{w}^{\varepsilon}\left(x-\left(v+\frac{\hbar}{2 m^{*}} q\right) \tau-\frac{B}{2} \tau^{2},\right. \\
v+B \tau, t-\tau), \\
\bar{D}_{2}^{B}:=\mathrm{e}^{-i \frac{\hbar}{2 m^{*}} q^{2} \tau} \bar{w}^{\varepsilon}\left(x-\left(v-\frac{\hbar}{2 m^{*}} q\right) \tau-\frac{B}{2} \tau^{2},\right. \\
v+B \tau, t-\tau) \\
-\mathrm{e}^{i \frac{\hbar}{2 m^{*}} q^{2} \tau} \bar{w}^{\varepsilon}\left(x-\left(v+\frac{\hbar}{2 m^{*}} q\right) \tau-\frac{B}{2} \tau^{2},\right. \\
\left.v+\frac{\hbar}{m^{*}} q+B \tau, t-\tau\right) .
\end{array}
$$

By integrating this equation with respect to $x$ we obtain after manipulations the Barker-Ferry equation [8]

$$
\begin{aligned}
\frac{\partial g^{\varepsilon}}{\partial t}- & B \cdot \nabla_{v} g^{\varepsilon} \\
= & \frac{2 \varepsilon^{2}}{\operatorname{Tr}} \int_{\tau=0}^{t} \int_{\mathbb{R}_{q}^{3}} \sum_{n=0}^{\infty} \sum_{\substack{k \in N^{n+1} \\
k_{1} \leq \cdots \leq k_{n+1}}} \sum_{j=1}^{n+1} \\
& \operatorname{Re}\left\{\mathrm{e}^{i\left(q \cdot v+\frac{\tau}{2} q \cdot B+\frac{\hbar}{2 m^{2}} q^{2}\right) \tau} R(q) \psi_{k_{j}}^{2}(q)\right.
\end{aligned}
$$

$$
\begin{gathered}
\left(\left(\mathrm{e}^{-\frac{i}{\hbar} \lambda_{k_{j}} \tau} \mathrm{e}^{-\beta \lambda_{\vec{k}}}+\mathrm{e}^{\frac{i}{\hbar} \lambda_{k_{j}} \tau} \mathrm{e}^{-\beta \lambda_{j} \vec{k}}\right)\right. \\
g^{\varepsilon}\left(v+\frac{\hbar}{2 m^{*}} q+B \tau, t-\tau\right) \\
-\left(\mathrm{e}^{\frac{i}{\hbar} \lambda_{k_{j}} \tau} \mathrm{e}^{-\beta \lambda_{\vec{k}}}+\mathrm{e}^{-\frac{i}{\hbar} \lambda_{k_{j}} \tau} \mathrm{e}^{-\beta \lambda_{j} \vec{k}}\right) \\
\left.\left.g^{\varepsilon}(v+B \tau, t-\tau)\right)\right\} d q d \tau
\end{gathered}
$$

where $g^{\varepsilon}(v, t):=\int_{\mathbb{R}_{x}^{3}} \bar{w}^{\varepsilon}(x, v, t) d x$.

\section{Acknowledgement}

The three authors acknowledge support from the joint NSF-DAAD grant 9603252 entitled "Asymptotische Methoden in der Quantenkinetik" and the NSF grant DMS 9706792.

\section{References}

[1] Argyres, P. N. (1992). Quantum Kinetic Equations for Electrons in High Electric and Phonon Fields, Phys. Lett. A, 171, 373-379.

[2] Barker, J. R. and Ferry, D. K. (1979). Self-Scattering PathVariable Formulation of High-Field, Time-Dependent, Quantum Kinetic Equations for Semiconductor Transport in the Finite-Collision-Duration Regime, Phys. Rev. Lett., 42, 1779-1781.

[3] Brunetti, R., Jacobini, C. and Rossi, F. (1989). Quantum theory of transient transport in semiconductors: A Monte Carlo approach, Phys. Rev. B, 39(19), 10781-10790.

[4] Landau, L. D. and Lifschitz, E. M. (1960). Lehrbuch der theoretischen Physik. Teil 3, Akademie Verlag, Berlin.

[5] Reed, M. and Simon, B. (1987). Methods of Modern Mathematical Physics I, Academic Press, 3rd edn.

[6] Reed, M. and Simon, B. (1986). Methods of Modern Mathematical Physics II, Academic Press, 6th edn.

[7] Reissland, J. A. (1973). The Physics of Phonons, John Wiley $\&$ Sons Ltd.

[8] Sano, N. and Yoshii, A., Quantum Kinetic Transport under High Electric Fields, Preprint.

[9] Ziman, J. M. (1960). Electrons and Phonons, Oxford University Press.

\section{Authors' Biographies}

Florian Frommlet is a Ph.D. student at the Technical University of Berlin. His research interests focus on the theory of quantum transport in semiconductor devices.

Peter A. Markowich received his Ph.D. in Applied Mathematics from the Technical University of Vienna (Austria) in 1980. He was Professor of 
Mathematics at the Technical University of Berlin (Germany) from 1989 to 1998 and is currently Proffessor of Mathematics at the University of Linz (Austria). His fields of interest are partial differential equations and kinetic theory.

Christian Ringhofer obtained his Ph.D. from the Polytechnical University of Vienna in 1981. From
1982 to 1984 he was on the faculty of the University of Wisconsin on a postdoctoral appointment. Since 1984 he has been on the faculty of Arizona State University. His interests include quantum mechanics, solid state transport theory, asymptotic methods and numerical methods for partial differential equations. 

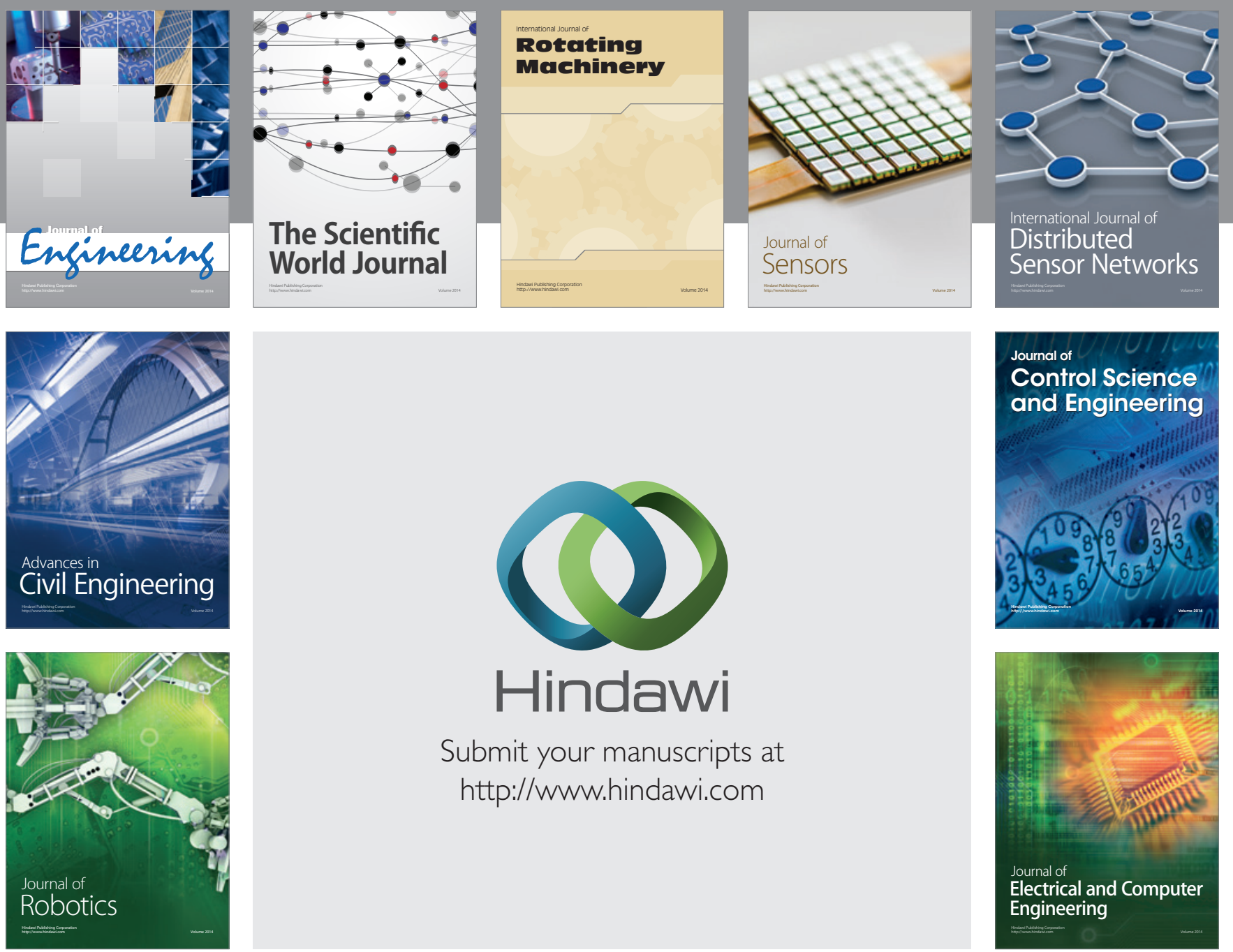

Submit your manuscripts at

http://www.hindawi.com
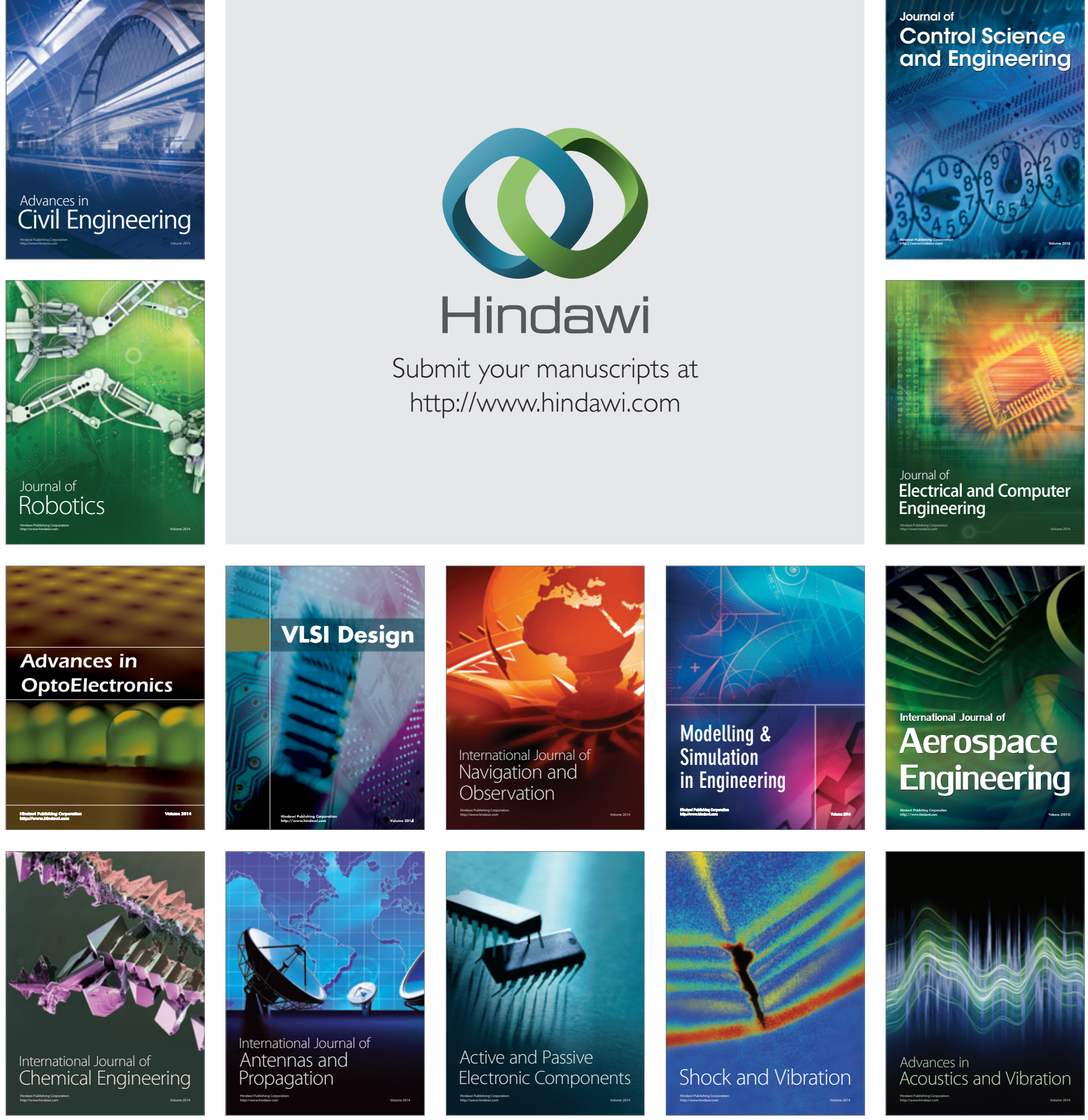